\title{
2 Less atmospheric radiative heating due to aspherical dust with coarser size
}

3 Akinori Ito $^{1 *}$, Adeyemi A. Adebiyi ${ }^{2}$, Yue Huang ${ }^{2}$, and Jasper F. Kok ${ }^{2}$

$4{ }^{1}$ Yokohama Institute for Earth Sciences, JAMSTEC, Yokohama, Kanagawa, 236-0001, Japan. ${ }^{2}$

90095, USA. *e-mail: akinorii@jamstec.go.jp

This document contains Supplementary Tables and Figures, which are summarized below:

- Table S1. Regionally averages of dust aerosol optical depth at $550 \mathrm{~nm}\left(\mathrm{DAOD}_{550}\right)$ in summer (June, July, and August).

- Table S2. Regionally averages of dust aerosol optical depth at $550 \mathrm{~nm}\left(\mathrm{DAOD}_{550}\right)$ in winter (December, January, and February), spring (March, April, and May), and autumn (September, October, and November).

- Table S3. Comparison of model estimates with semi-observation-based data of dust clear-sky SW radiative effect efficiency at the surface $\left(\mathrm{W} \cdot \mathrm{m}^{-2} \mathrm{DAOD}{ }^{-1}\right)$.

- Table S4. Comparison of model estimates with semi-observation-based data of dust clear-sky SW radiative effect efficiency at TOA $\left(\mathrm{W} \cdot \mathrm{m}^{-2} \mathrm{DAOD}{ }^{-1}\right)$.

- Table S5. Comparison of model estimates with semi-observation-based data of dust clear-sky LW radiative effect efficiency at the surface $\left(\mathrm{W} \cdot \mathrm{m}^{-2} \mathrm{DAOD}{ }^{-1}\right)$.

- Table S6. Comparison of model estimates with semi-observation-based data of dust clear-sky LW radiative effect efficiency at TOA $\left(\mathrm{W} \cdot \mathrm{m}^{-2} \mathrm{DAOD}{ }^{-1}\right)$.

- Figure S1. Comparison of semi-observation-based and modeled DAOD ${ }_{550}$ in summer. Results were shown for default (blue square), improved (red circles), and fine-global (black triangles) simulations. The dashed line represented a 1: 1 correspondence. The summer season was shown because most semi-observation-based data on dust radiative effects were available. The comparison for other seasons was presented in Table S2.

- Figure S2. Comparison of semi-observation-based and modeled dust SW radiative effect efficiency in summer. Results were shown at the surface (red circles) and TOA (blue square) from (a) default, (b) improved, (c) fine-global, (d) coarse-region, (e) coarse-mineral, (f) coarse-absorption simulations. The dashed line represented a $1: 1$ correspondence. The correlation coefficients $(r)$ and root mean square errors (RMSE) were also shown. The regionally averaged values were listed in Tables S3 and S4 at the surface and TOA, respectively.

- Figure S3. Summertime dust SW radiative effect efficiency. The model results were shown (a) and (b) fine-global, (c) and (d) coarse-region, (e) and (f) coarse-mineral, and (g) and (h) coarse-mineral coarse-absorption simulations at the surface and TOA, respectively. The regionally averaged values were listed in Tables S3 and S4 at the surface and TOA, respectively.

- Figure S4. Comparison of semi-observation-based and modeled dust LW radiative effect efficiency in summer. Results were shown at the surface (red circles) and TOA (blue square) from (a) default, (b) improved, (c) fine-global, (d) coarse-global, (e) coarse-region, (f) coarse-absorption. The dashed line represented a $1: 1$ correspondence. The correlation coefficients $(r)$ and root mean square errors (RMSE) were also shown. The regionally averaged values were listed in Tables S5 and S6 at the surface and TOA, respectively.

- Figure S5. Summertime dust LW radiative effect efficiency. The model results were shown (a) and (b) fine-global, (c) and (d) coarse-global, (e) and (f) coarse-region, and (g) and (h) coarse-absorption simulations at the surface and TOA, respectively. The regionally averaged values were listed in Tables S5 and S6 at the surface and TOA, respectively. 
Table S1. Regionally averages of dust aerosol optical depth at $550 \mathrm{~nm}\left(\mathrm{DAOD}_{550}\right)$ in summer (June, July, and August).

\begin{tabular}{|c|c|c|c|c|c|}
\hline Region name & Region coordinates & Semi-observation-based data & Default simulation & Improved simulation & Fine-global simulation \\
\hline Mid-Atlantic & $4^{\circ}-40^{\circ} \mathrm{N}, 50^{\circ}-20^{\circ} \mathrm{W}$ & $0.143 \pm 0.005$ & 0.094 & 0.083 & 0.148 \\
\hline African West Coast & $10^{\circ}-34^{\circ} \mathrm{N}, 20^{\circ}-5^{\circ} \mathrm{W}$ & $0.365 \pm 0.016$ & 0.357 & 0.294 & 0.563 \\
\hline Northern Africa & $26^{\circ}-40^{\circ} \mathrm{N}, 5^{\circ} \mathrm{W}-30^{\circ} \mathrm{E}$ & $0.207 \pm 0.016$ & 0.197 & 0.194 & 0.310 \\
\hline Mali/Niger & $10^{\circ}-26^{\circ} \mathrm{N}, 5^{\circ} \mathrm{W}-10^{\circ} \mathrm{E}$ & $0.462 \pm 0.044$ & 0.379 & 0.397 & 0.597 \\
\hline Bodele/Sudan & $10^{\circ}-26^{\circ} \mathrm{N}, 10^{\circ}-40^{\circ} \mathrm{E}$ & $0.310 \pm 0.018$ & 0.297 & 0.349 & 0.469 \\
\hline Northern Middle East & $26^{\circ}-40^{\circ} \mathrm{N}, 30^{\circ}-50^{\circ} \mathrm{E}$ & $0.164 \pm 0.015$ & 0.209 & 0.168 & 0.327 \\
\hline Southern Middle East & $0^{\circ}-26^{\circ} \mathrm{N}, 40^{\circ}-67.5^{\circ} \mathrm{E}$ & $0.330 \pm 0.044$ & 0.438 & 0.384 & 0.691 \\
\hline Kyzyl Kum & $26^{\circ}-50^{\circ} \mathrm{N}, 50^{\circ}-67.5^{\circ} \mathrm{E}$ & $0.154 \pm 0.034$ & 0.307 & 0.201 & 0.481 \\
\hline Thar & $20^{\circ}-50^{\circ} \mathrm{N}, 67.5^{\circ}-75^{\circ} \mathrm{E}$ & $0.319 \pm 0.029$ & 0.167 & 0.156 & 0.265 \\
\hline Taklamakan & $30^{\circ}-50^{\circ} \mathrm{N}, 75^{\circ}-92.5^{\circ} \mathrm{E}$ & $0.171 \pm 0.026$ & 0.040 & 0.094 & 0.064 \\
\hline Gobi & $36^{\circ}-50^{\circ} \mathrm{N}, 92.5^{\circ}-115^{\circ} \mathrm{E}$ & $0.102 \pm 0.035$ & 0.032 & 0.117 & 0.051 \\
\hline North America & $20^{\circ}-45^{\circ} \mathrm{N}, 80^{\circ}-130^{\circ} \mathrm{W}$ & $0.028 \pm 0.010$ & 0.010 & $\mathbf{0 . 0 3 0}$ & 0.016 \\
\hline South America & $0^{\circ}-55^{\circ} \mathrm{S}, 80^{\circ}-55^{\circ} \mathrm{W}$ & $0.010 \pm 0.006$ & 0.009 & 0.013 & 0.015 \\
\hline Southern Africa & $10^{\circ}-35^{\circ} \mathrm{S}, 10^{\circ}-40^{\circ} \mathrm{E}$ & $0.013 \pm 0.005$ & 0.014 & 0.020 & 0.022 \\
\hline Australia & $10^{\circ}-40^{\circ} \mathrm{S}, 110^{\circ}-160^{\circ} \mathrm{E}$ & $0.010 \pm 0.005$ & 0.005 & 0.013 & 0.008 \\
\hline Correlation coefficients & & & 0.86 & 0.91 & 0.86 \\
\hline Root mean square errors & & & 0.08 & 0.06 & 0.16 \\
\hline
\end{tabular}

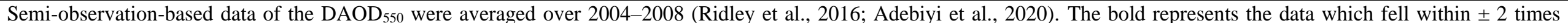
standard deviation of the measurements. The correlation coefficients and root mean square errors were also shown. The scatter plot was shown in Fig. S1. 
Table S2. Regionally averages of dust aerosol optical depth at $550 \mathrm{~nm}$ (DAOD 550 ) in winter (December, January, and February), spring (March, April, and May), and autumn (September, October, and November).

\begin{tabular}{|c|c|c|c|c|c|c|c|c|c|}
\hline & Winter & & & Spring & & & Autumn & & \\
\hline Region name & Semi-observation-based & Default & Improved & Semi-observation-based & Default & Improved & Semi-observation-based & Default & Improved \\
\hline Mid-Atlantic & $0.064 \pm 0.013$ & 0.045 & 0.072 & $0.106 \pm 0.008$ & 0.026 & 0.034 & $0.084 \pm 0.006$ & 0.024 & 0.027 \\
\hline African West Coast & $0.180 \pm 0.010$ & 0.129 & 0.223 & $0.250 \pm 0.019$ & 0.127 & 0.145 & $0.233 \pm 0.022$ & 0.132 & 0.149 \\
\hline Northern Africa & $0.118 \pm 0.011$ & 0.029 & 0.108 & $0.219 \pm 0.010$ & 0.174 & 0.200 & $0.151 \pm 0.016$ & 0.092 & 0.134 \\
\hline Mali/Niger & $0.257 \pm 0.019$ & 0.215 & 0.377 & $0.441 \pm 0.022$ & 0.341 & 0.358 & $0.277 \pm 0.023$ & 0.247 & 0.268 \\
\hline Bodele/Sudan & $0.191 \pm 0.006$ & 0.149 & 0.360 & $0.339 \pm 0.023$ & 0.34 & 0.406 & $0.212 \pm 0.021$ & 0.223 & 0.276 \\
\hline Northern Middle East & $0.112 \pm 0.011$ & 0.038 & 0.165 & $0.223 \pm 0.011$ & 0.169 & 0.226 & $0.113 \pm 0.019$ & 0.115 & 0.133 \\
\hline Southern Middle East & $0.123 \pm 0.018$ & 0.056 & 0.144 & $0.204 \pm 0.021$ & 0.096 & 0.176 & $0.150 \pm 0.020$ & 0.157 & 0.135 \\
\hline Kyzyl Kum & $0.115 \pm 0.017$ & 0.028 & 0.116 & $0.176 \pm 0.026$ & 0.096 & 0.204 & $0.101 \pm 0.018$ & 0.129 & 0.138 \\
\hline Thar & $0.130 \pm 0.029$ & 0.024 & 0.094 & $0.238 \pm 0.033$ & 0.091 & 0.173 & $0.135 \pm 0.037$ & 0.072 & 0.072 \\
\hline Taklamakan & $0.119 \pm 0.013$ & 0.008 & 0.029 & $0.275 \pm 0.027$ & 0.03 & 0.110 & $0.104 \pm 0.011$ & 0.016 & 0.045 \\
\hline Gobi & $0.093 \pm 0.022$ & 0.006 & 0.028 & $0.192 \pm 0.022$ & 0.053 & 0.183 & $0.047 \pm 0.021$ & 0.018 & 0.091 \\
\hline North America & $0.010 \pm 0.005$ & 0.003 & 0.027 & $0.029 \pm 0.011$ & 0.014 & 0.031 & $0.012 \pm 0.006$ & 0.006 & 0.014 \\
\hline South America & $0.019 \pm 0.011$ & 0.024 & 0.016 & $0.013 \pm 0.007$ & 0.013 & 0.011 & $0.016 \pm 0.009$ & 0.022 & 0.013 \\
\hline Southern Africa & $0.016 \pm 0.007$ & 0.02 & 0.011 & $0.011 \pm 0.005$ & 0.009 & 0.009 & $0.016 \pm 0.007$ & 0.028 & 0.012 \\
\hline Australia & $0.025 \pm 0.013$ & 0.016 & 0.028 & $0.013 \pm 0.006$ & 0.008 & 0.012 & $0.023 \pm 0.011$ & 0.020 & 0.020 \\
\hline Correlation coefficients & & 0.83 & 0.89 & & 0.84 & 0.89 & & 0.88 & 0.88 \\
\hline Root mean square errors & & 0.06 & 0.06 & & 0.10 & 0.06 & & 0.05 & 0.04 \\
\hline
\end{tabular}

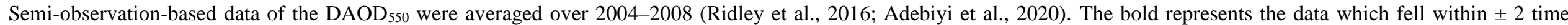
standard deviation of the measurements. The correlation coefficients and root mean square errors were also shown. 
Table S3. Comparison of model estimates with semi-observation-based data of dust clear-sky SW radiative effect efficiency at the surface $\left(\mathrm{W}^{-} \mathrm{m}^{-2} \mathrm{DAOD}{ }^{-1}\right)$.

\begin{tabular}{|c|c|c|c|c|c|c|c|c|c|}
\hline Number & Month & Semi-observation-based & Default & Improved & Fine-global & Coarse-global & Coarse-region & Coarse-mineral & Coarse-absorption \\
\hline 2 & $6,7,8$ & $-65^{\mathrm{a}}$ & -63 & -51 & -43 & -51 & -49 & -68 & -39 \\
\hline 3 & $6,7,8$ & $-86^{\mathrm{b}}$ & -69 & -56 & -47 & -56 & -53 & -74 & -43 \\
\hline 12 & 9 & $69^{c}$ & -58 & -45 & -39 & -45 & -44 & -60 & -34 \\
\hline 14 & $4,5,6$ & $-60^{\mathrm{d}}$ & -56 & -44 & -41 & -44 & -42 & -53 & -33 \\
\hline
\end{tabular}

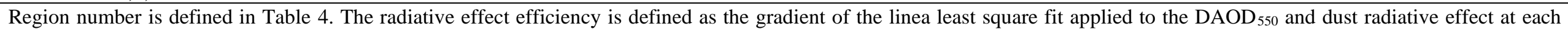
grid. The area weighted averages for land or ocean are listed. ${ }^{\mathrm{a}} \mathrm{Li}$ et al. (2004). b'Song et al. (2018). ${ }^{\mathrm{d} D i}$ Biagio et al. (2010). ${ }^{\mathrm{e}} \mathrm{Hansell}$ et al. (2012). 
Table S4. Comparison of model estimates with semi-observation-based data of dust clear-sky SW radiative effect efficiency at TOA (W· $\mathrm{m}^{-2} \mathrm{DAOD}{ }^{-1}$ ).

\begin{tabular}{|c|c|c|c|c|c|c|c|c|c|}
\hline Number & Month & Semi-observation-based & Default & Improved & Fine-global & Coarse-global & Coarse-region & Coarse-mineral & Coarse-absorption \\
\hline 1 & $6,7,8$ & Near $0^{\mathrm{a}}$ & 17 & 13 & 3 & 13 & 14 & 28 & -1 \\
\hline 2 & $6,7,8$ & $-35 \pm 3^{b}$ & -13 & -12 & -18 & -12 & -13 & -0.3 & -20 \\
\hline 3 & $6,7,8$ & $-27^{\mathrm{c}}$ & -16 & -15 & -21 & -15 & -16 & -0.7 & -24 \\
\hline 4 & $6,7,8$ & $-48 \pm 4^{\mathrm{d}}$ & -20 & -17 & -21 & -17 & -17 & -6.9 & -23 \\
\hline 12 & 9 & $-46^{\mathrm{e}}$ & -22 & -16 & -22 & -16 & -16 & -9.0 & -20 \\
\hline
\end{tabular}

Region number is defined in Table 4. The radiative effect efficiency is defined as the gradient of the linea least square fit applied to the DAOD 550 and dust radiative effect at each

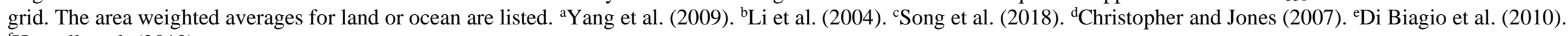

${ }^{\mathrm{f}}$ Hansell et al. (2012). 
Table S5. Comparison of model estimates with semi-observation-based data of dust clear-sky LW radiative effect efficiency at the surface $\left(\mathrm{W}^{-\mathrm{m}^{-2}} \mathrm{DAOD}{ }^{-1}\right)$.

\begin{tabular}{|c|c|c|c|c|c|c|c|c|c|}
\hline Number & Month & Semi-observation-based data & Default & Improved & Fine-global & Coarse-global & Coarse-region & Coarse-mineral & Coarse-absorption \\
\hline 3 & $6,7,8$ & $24^{\mathrm{a}}$ & 8.8 & 21 & 7.5 & 12 & 13 & 21 & 16 \\
\hline 13 & 9 & $16^{\mathrm{b}}$ & 16 & 30 & 13 & 18 & 19 & 30 & 24 \\
\hline 14 & $4,5,6$ & $31-35^{\mathrm{c}}$ & 22 & 33 & 18 & 20 & 22 & 33 & 25 \\
\hline
\end{tabular}

Region number is defined in Table 4 . The radiative effect efficiency is defined as the gradient of the linea least square fit

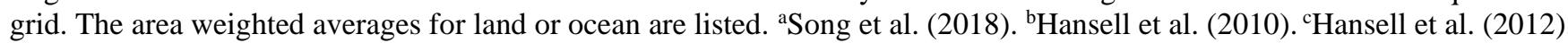


Table S6. Comparison of model estimates with semi-observation-based data of dust clear-sky LW radiative effect efficiency at TOA $\left(\mathrm{W}^{-\mathrm{m}^{-2}} \mathrm{DAOD}{ }^{-1}\right)$.

\begin{tabular}{|c|c|c|c|c|c|c|c|c|c|}
\hline Number & Month & Semi-observation-based data & Default & Improved & Fine-global & Coarse-global & Coarse-region & Coarse-mineral & Coarse-absorption \\
\hline 1 & $6,7,8$ & $24 \pm 1.3^{\mathrm{a}}$ & 8.3 & 19.9 & 7.2 & 11.0 & 10.8 & 19.9 & 14.5 \\
\hline 3 & $6,7,8$ & $9.5^{\mathrm{b}}$ & 4.3 & 11.6 & 3.8 & 5.8 & 6.1 & 11.6 & 8.1 \\
\hline 4 & $6,7,8$ & $9.0 \pm 3.5^{\mathrm{c}}$ & 3.5 & 9.7 & 3.1 & 4.9 & 5.1 & 9.7 & 6.9 \\
\hline 5 & $6,7,8$ & $22^{\mathrm{d}}$ & 6.8 & 16.1 & 5.8 & 8.8 & 8.6 & 16.1 & 11.6 \\
\hline 5 & 9 & $15^{\mathrm{e}}$ & 5.5 & 11.7 & 4.8 & 6.2 & 6.0 & 11.7 & 8.4 \\
\hline 6 & $6,7,8$ & $17^{\mathrm{d}}$ & 7.6 & 20.3 & 6.6 & 11.1 & 11.6 & 20.3 & 14.5 \\
\hline 6 & 9 & $20^{\mathrm{e}}$ & 7.3 & 16.1 & 6.4 & 8.5 & 8.9 & 16.1 & 11.5 \\
\hline 7 & $6,7,8$ & $16^{\mathrm{d}}$ & 9.1 & 21.0 & 7.9 & 11.9 & 11.9 & 21.0 & 15.6 \\
\hline 7 & 9 & $21^{\mathrm{e}}$ & 6.3 & 14.5 & 5.5 & 8.1 & 7.4 & 14.5 & 10.7 \\
\hline 8 & $6,7,8$ & $21^{\mathrm{d}}$ & 5.7 & 13.5 & 5.0 & 7.3 & 6.8 & 13.5 & 9.8 \\
\hline 8 & 9 & $19^{\mathrm{e}}$ & 5.1 & 8.7 & 4.4 & 4.7 & 4.4 & 8.7 & 6.4 \\
\hline 9 & $6,7,8$ & $25^{\mathrm{d}}$ & 5.1 & 11.7 & 4.5 & 5.9 & 5.5 & 11.7 & 8.2 \\
\hline 9 & 9 & $1^{\mathrm{e}}$ & 4.0 & 7.4 & 3.6 & 3.7 & 3.3 & 7.4 & 5.2 \\
\hline 10 & $6,7,8$ & $20^{\mathrm{d}}$ & 6.6 & 14.1 & 5.8 & 7.2 & 6.9 & 14.1 & 9.9 \\
\hline 10 & 9 & $11^{\mathrm{e}}$ & 5.5 & 10.5 & 4.8 & 5.5 & 5.0 & 10.5 & 7.5 \\
\hline 11 & $6,7,8$ & $18^{\mathrm{d}}$ & 8.4 & 19.0 & 7.4 & 9.9 & 9.1 & 19.0 & 13.5 \\
\hline 11 & 9 & $11^{\mathrm{e}}$ & 6.1 & 10.3 & 5.4 & 5.2 & 4.8 & 10.3 & 7.3 \\
\hline 13 & 9 & $13^{\mathrm{f}}$ & 4.4 & 30 & 3.8 & 18 & 19 & 30 & 24 \\
\hline 14 & $4,5,6$ & $17-21^{\mathrm{g}}$ & 4.4 & 9.0 & 3.8 & 4.6 & 5.0 & 9.0 & 6.1 \\
\hline
\end{tabular}

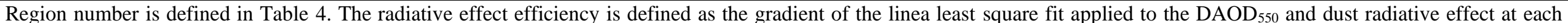

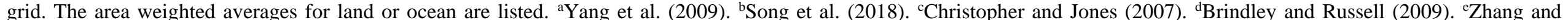
Christopher (2003). ${ }^{\mathrm{f}}$ Hansell et al. (2010). ${ }^{\mathrm{g}} \mathrm{Hansell}$ et al. (2012). 


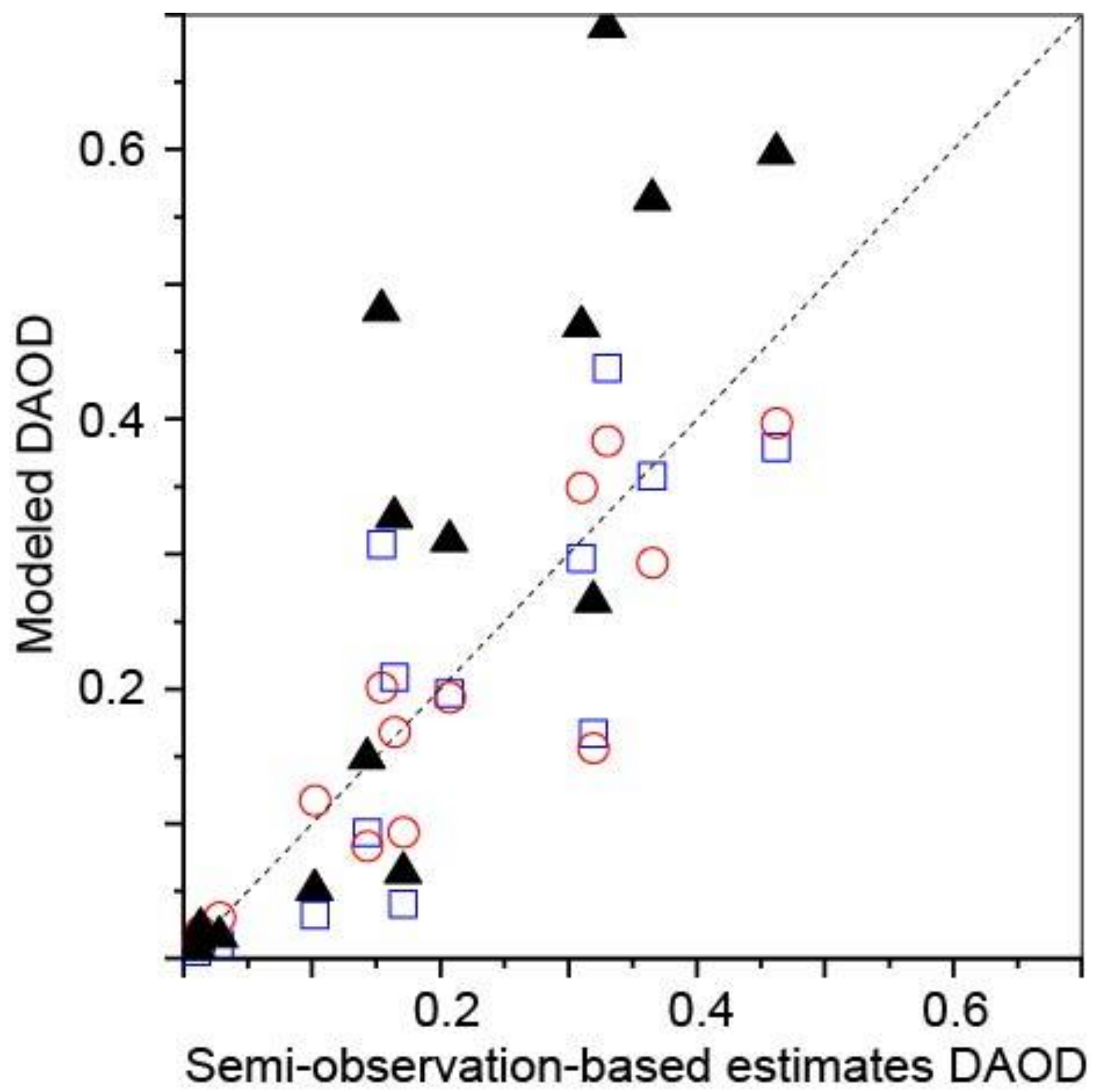

$\frac{1}{2}$

Figure S1. Comparison of semi-observation-based and modeled $\mathrm{DAOD}_{550}$ in summer. Results were shown for

3 default (blue square), improved (red circles), and fine-global (black triangles) simulations. The dashed line

4 represented a $1: 1$ correspondence. The summer season was shown because most semi-observation-based data on

5 dust radiative effects were available. The comparison for other seasons was presented in Table S2. 
(a) Experiment 1

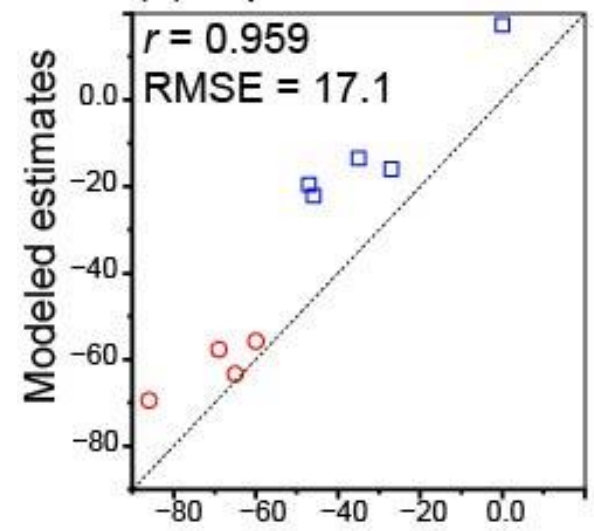

(d) Experiment 5

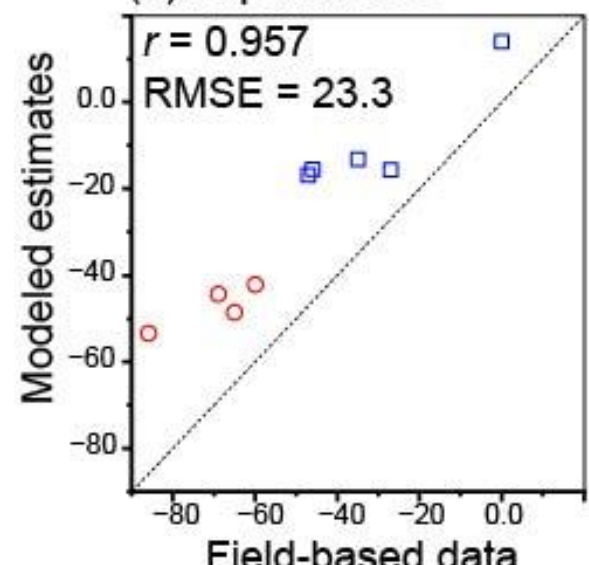

(b) Experiment 2

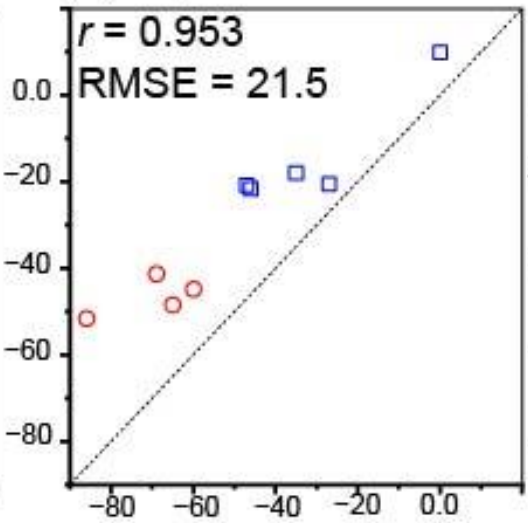

(e) Experiment 6

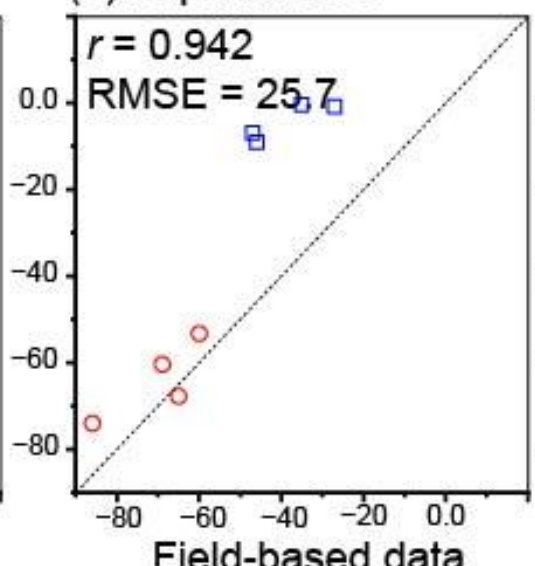

(c) Experiment 3

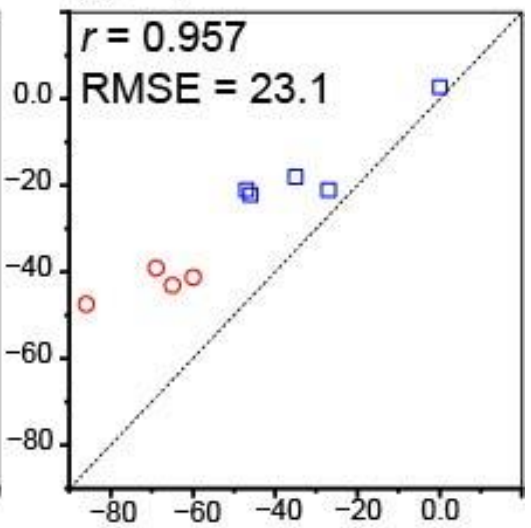

(f) Experiment 7

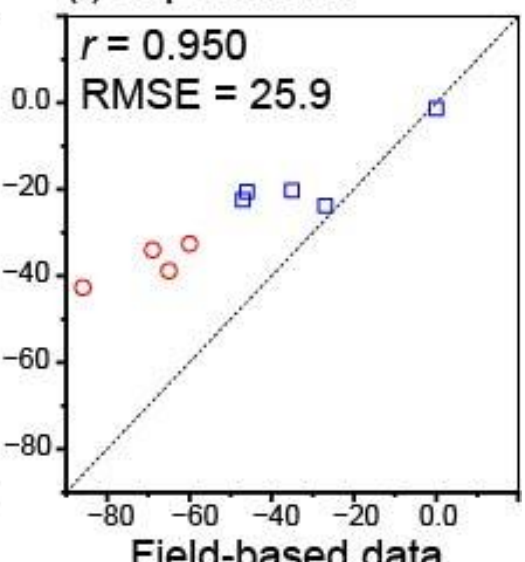

Figure S2. Comparison of semi-observation-based and modeled dust SW radiative effect efficiency in summer.

8 Results were shown at the surface (red circles) and TOA (blue square) from (a) default, (b) improved, (c) fine-global,

9 (d) coarse-region, (e) coarse-mineral, (f) coarse-absorption simulations. The dashed line represented a $1: 1$

10 correspondence. The correlation coefficients $(r)$ and root mean square errors (RMSE) were also shown. The

11 regionally averaged values were listed in Tables S3 and S4 at the surface and TOA, respectively. 
SW at the surface

(a) Experiment 3

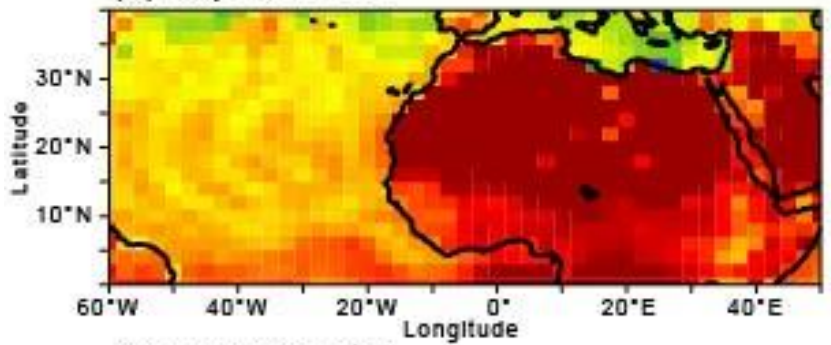

(c) Experiment 5

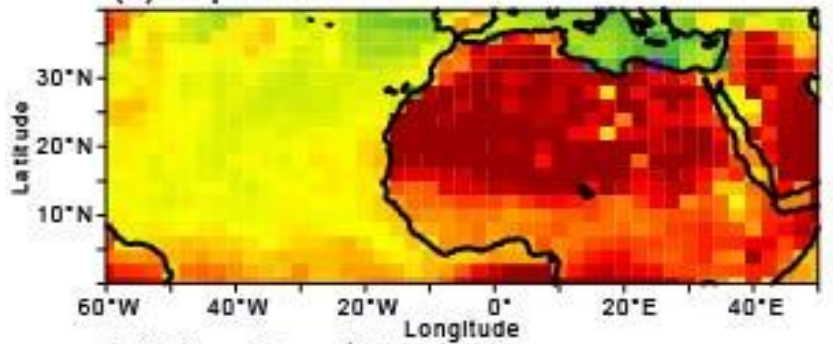

(e) Experiment 6

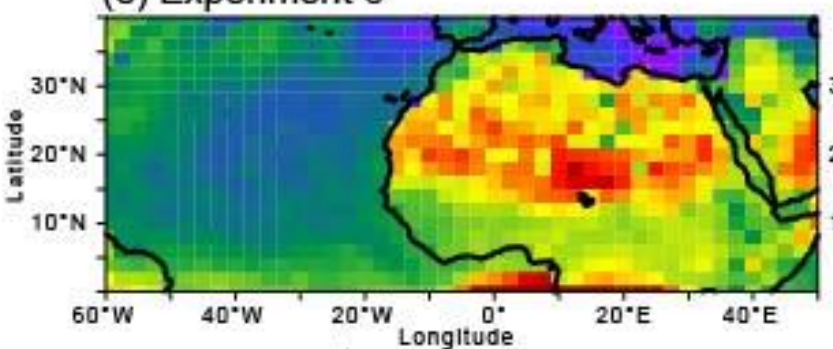

(g) Experiment 7

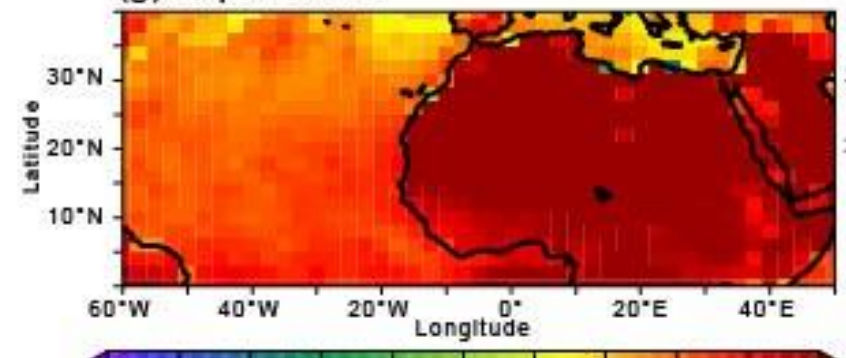

$-90-85-80-75-70-65-60-55-50-45-4$

\section{Radiative effect efficiency $\left(\mathrm{W} \mathrm{m}^{-2}\right)$}

13 Figure S3. Summertime dust SW radiative effect efficiency. The model results were shown (a) and (b) fine-global,

14 (c) and (d) coarse-region, (e) and (f) coarse-mineral, and (g) and (h) coarse-mineral coarse-absorption simulations at

SW at the TOA

(b) Experiment 3

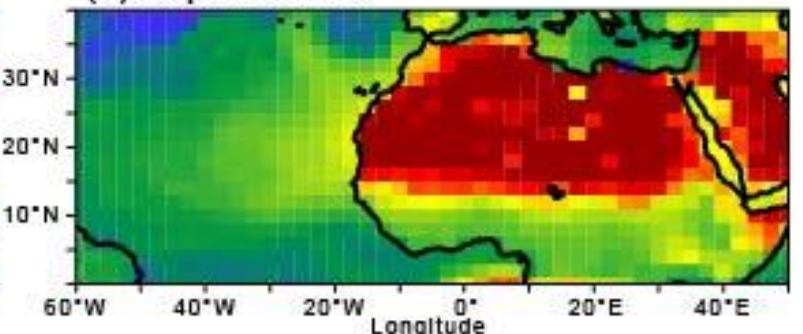

(d) Experiment 5

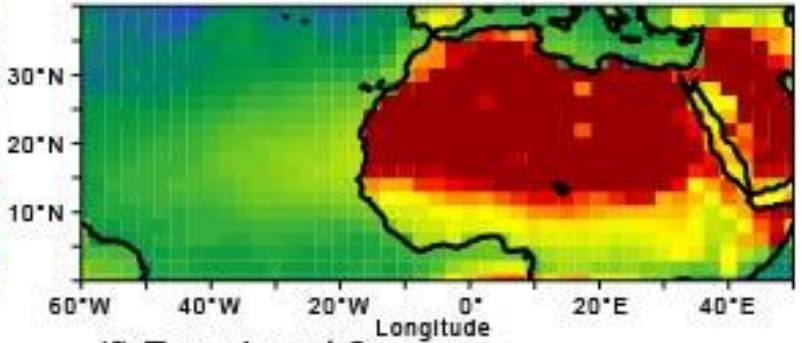

(f) Experiment 6

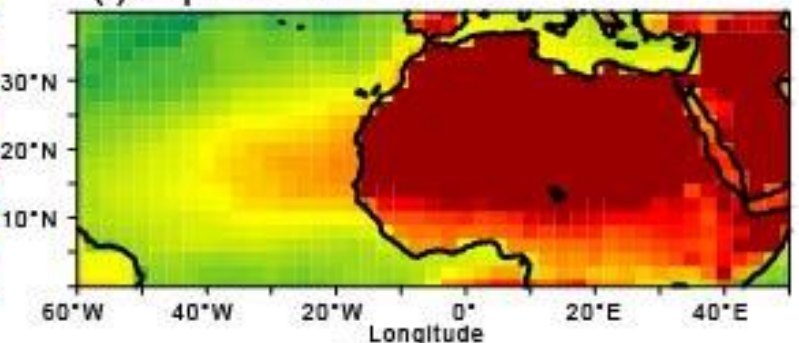

(h) Experiment 7
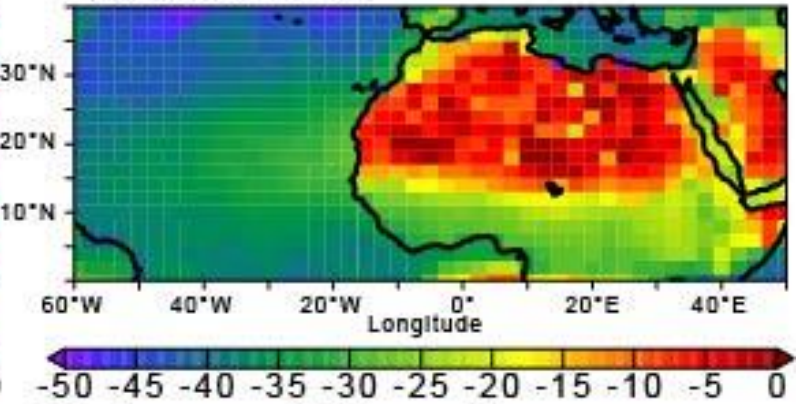
(a) Experiment 1

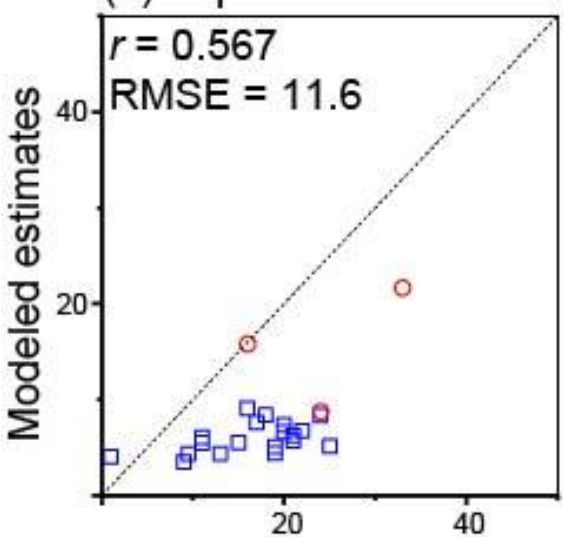

(d) Experiment 4

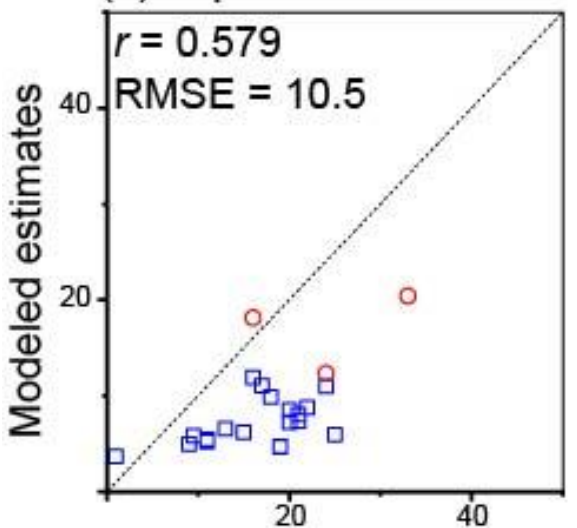

Field-based data (b) Experiment 2

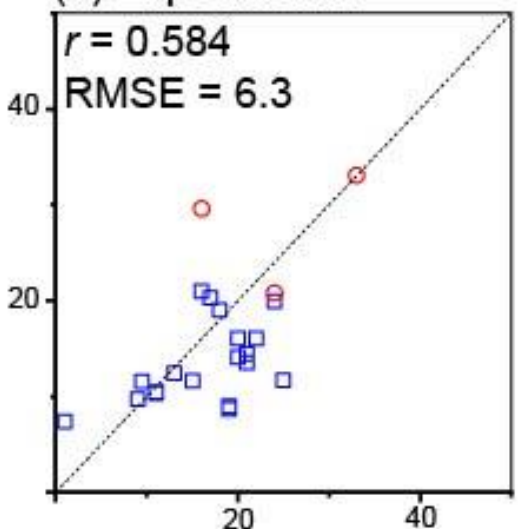

(e) Experiment 5

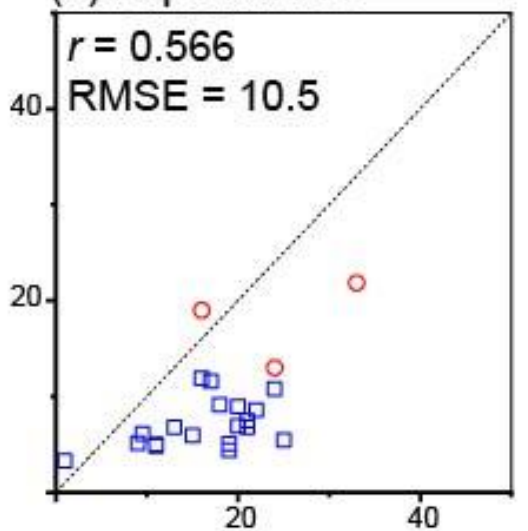

Field-based data (c) Experiment 3

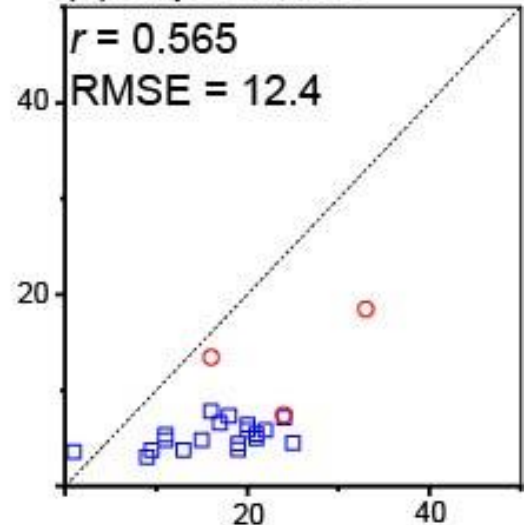

(f) Experiment 7

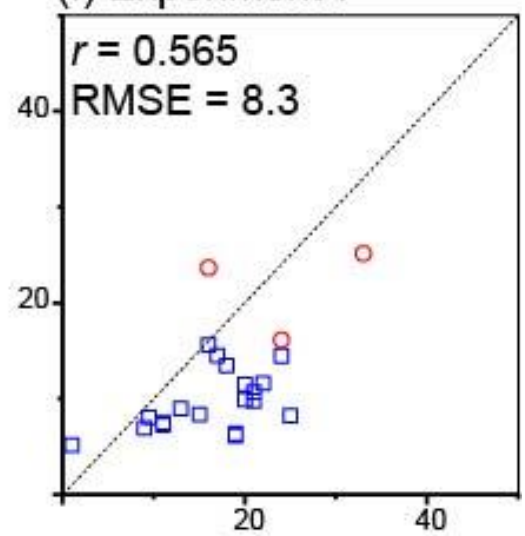

Field-based data

17 Figure S4. Comparison of semi-observation-based and modeled dust LW radiative effect efficiency in summer.

19 Results were shown at the surface (red circles) and TOA (blue square) from (a) default, (b) improved, (c) fine-global,

20 (d) coarse-global, (e) coarse-region, (f) coarse-absorption. The dashed line represented a $1: 1$ correspondence. The

21 correlation coefficients $(r)$ and root mean square errors (RMSE) were also shown. The regionally averaged values were listed in Tables S5 and S6 at the surface and TOA, respectively. 
LW at the surface

(a) Experiment 3

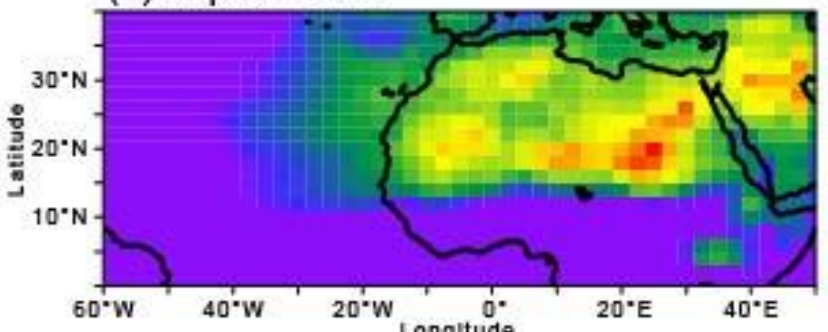

(c) Experiment 4

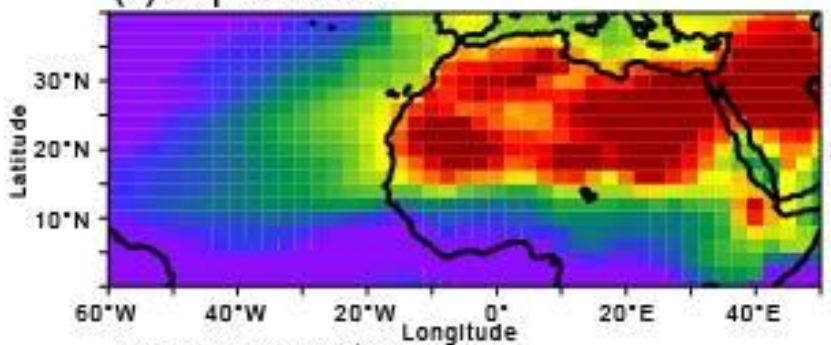

(e) Experiment 5

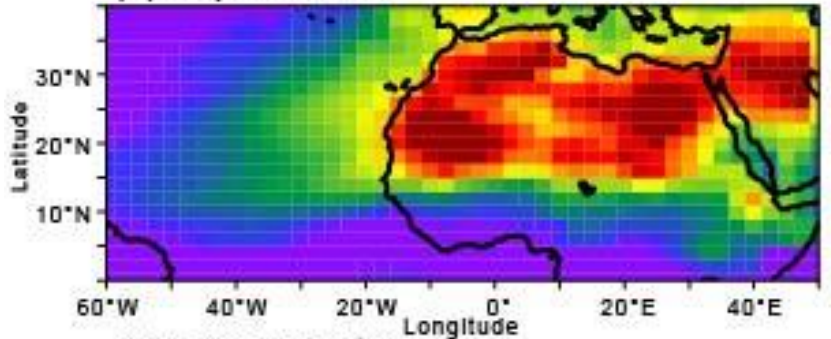

(g) Experiment 7

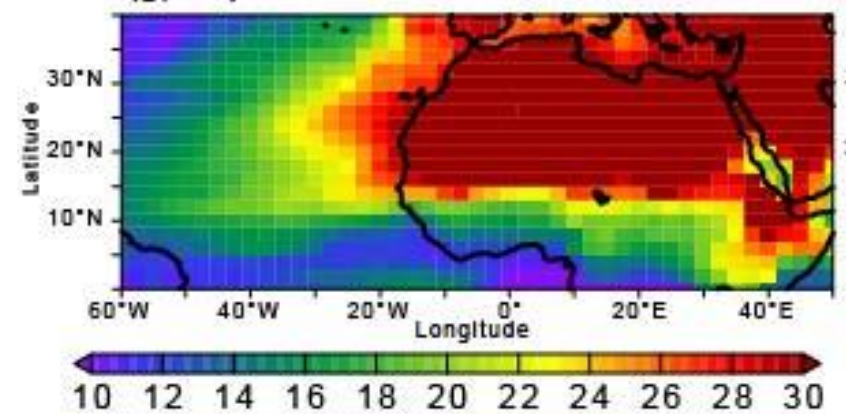

LW at the TOA

(b) Experiment 3

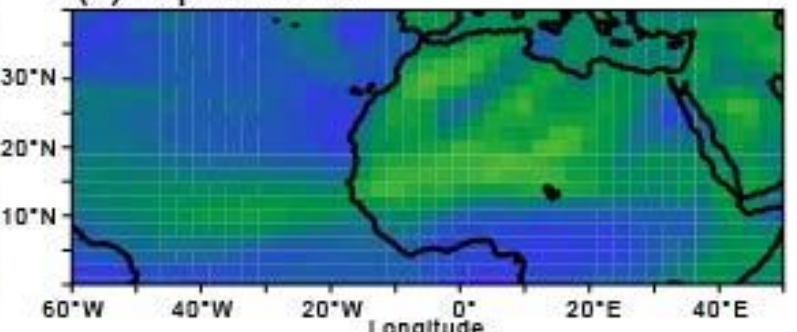

(d) Experiment 4

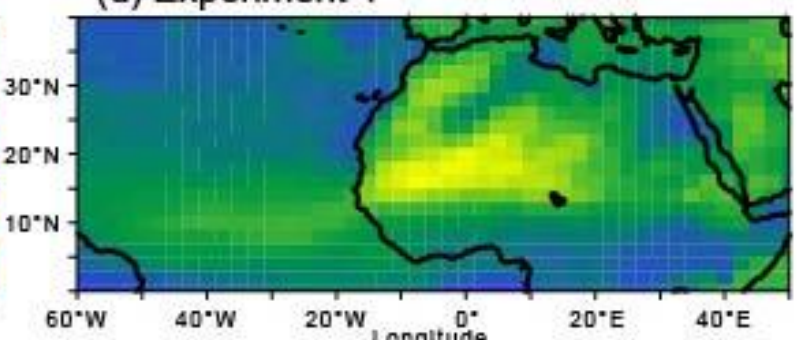

(f) Experiment 5

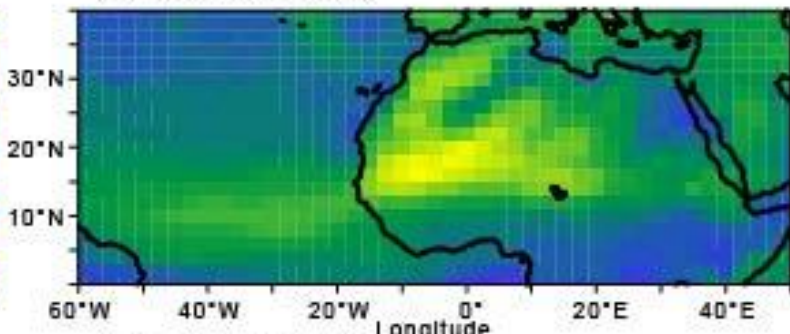

(h) Experiment 7
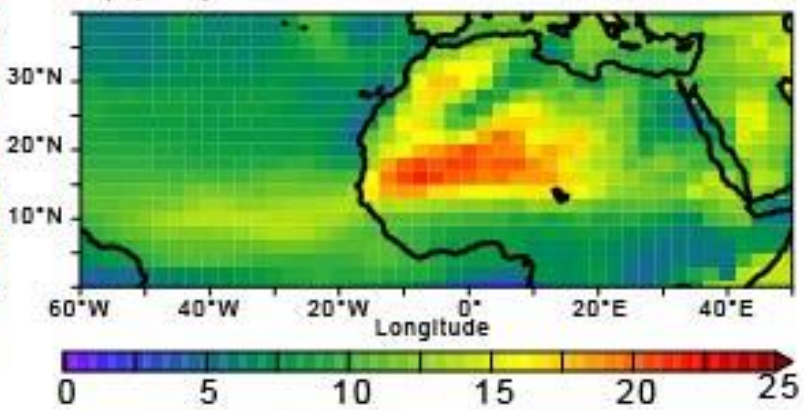
Radiative effect efficiency $\left(\mathrm{W} \mathrm{m}^{-2}\right)$

24 Figure S5. Summertime dust LW radiative effect efficiency. The model results were shown (a) and (b) fine-global,

25 (c) and (d) coarse-global, (e) and (f) coarse-region, and (g) and (h) coarse-absorption simulations at the surface and

26 TOA, respectively. The regionally averaged values were listed in Tables S5 and S6 at the surface and TOA,

27 respectively. 